\title{
T cell receptor clonotypes modulate the protective effect of HLA class I alleles in HIV-1 infection
}

\author{
Huabiao Chen ${ }^{1,2,7}$, Zaza M. Ndhlovu1,2,7, Dongfang Liu1, Lindsay C. Porter ${ }^{1}$, Justin W. \\ Fang $^{1}$, Sam Darko ${ }^{3}$, Mark A. Brockman ${ }^{1,4}$, Toshiyuki Miura ${ }^{1,5}$, Zabrina L. Brumme ${ }^{1,4}$, Arne \\ Schneidewind ${ }^{1,6}$, Alicja Piechocka-Trocha ${ }^{1,2}$, Kevin T. Cesa ${ }^{1}$, Jennifer Sela ${ }^{1}$, Thai D. \\ Cung ${ }^{1}$, Ildiko Toth ${ }^{1}$, Florencia Pereyra ${ }^{1}$, Xu G. Yu${ }^{1}$, Daniel C. Douek ${ }^{3}$, Daniel E. Kaufmann ${ }^{1}$, \\ Todd M. Allen ${ }^{1}$, and Bruce D. Walker ${ }^{1,2}$ \\ ${ }^{1}$ Ragon Institute of Massachusetts General Hospital, Massachusetts Institute of Technology, and \\ Harvard University, Boston, MA 02114, USA \\ ${ }^{2}$ Howard Hughes Medical Institute, Chevy Chase, MD 20815, USA \\ ${ }^{3}$ Human Immunology Section, Vaccine Research Center, National Institute of Allergy and \\ Infectious Diseases, National Institute of Health, Bethesda, MD 20892, USA \\ ${ }^{4}$ Simon Fraser University, Burnaby, BC V5A 1S6, Canada \\ 5 Institute of Medical Science, University of Tokyo, Tokyo 108-8639, Japan \\ ${ }^{6}$ Department of Internal Medicine, University Hospital Regensburg, 94053 Regensburg, Germany
}

\section{Abstract}

Human leukocyte antigen (HLA) B*27 and B*57 are associated with protection against HIV-1 disease progression, yet most persons expressing these alleles are unable to control HIV-1. Here we show that HLA-B $* 27$-restricted $\mathrm{CD} 8^{+} \mathrm{T}$ cells in controllers and progressors differ in their ability to inhibit virus replication through targeting of the immunodominant Gag epitope. This is associated with distinct TCR clonotypes, characterized by superior control of HIV-1 replication in vitro, greater cross-reactivity against epitope variants, and enhanced perforin delivery. Clonotypespecific differences in antiviral efficacy were also observed for an immunodominant HLA-B*57 restricted response in controllers and progressors. Thus, the efficacy of protective alleles is modulated by specific TCR clonotypes selected in natural infection, providing a functional explanation for divergent HIV-1 outcomes.

A subset of HIV-1-infected persons, here termed elite controllers, are distinguished by their ability to maintain a state of apparent durable control of HIV-1 replication without the need for antiviral therapy ${ }^{1,2}$. Viral control is linked to expression of certain human leukocyte antigen (HLA) class I alleles ${ }^{3-5}$, particularly $\mathrm{B} * 57, \mathrm{~B} * 27$, and $\mathrm{B} * 5801$, suggesting an immunologic basis related to $\mathrm{CD} 8^{+} \mathrm{T}$ cell function. Indeed, a recent genome-wide

\footnotetext{
Correspondence should be addressed to: Bruce D. Walker (bwalker@partners.org).

${ }^{7} \mathrm{HC}$ and $\mathrm{ZMN}$ contributed equally to this work.
}

\section{AUTHOR CONTRIBUTIONS}

H.C. was responsible for the overall conduct of the study, under the supervision of B.D.W.; H.C., Z.M.N., and B.D.W. contributed to the experimental design; H.C., Z.M.N., L.C.P., J.W.F., S.D., T.M., Z.L.B., K.T.C. and J.S. performed the experiments; M.A.B., A.S. and T.M.A. constructed HIV-1 variants and GXR cell lines; D.L. and D.E.K. performed the imaging expriments; T.D.C. and X.G.Y. helped TCR sequencing; F.P., A.P. and I.T. provided clinical samples; H.C., Z.M.N., and B.D.W. wrote the paper, and all authors contributed to revisions.

COMPETING INTERESTS STATEMENT

The authors declare that they have no competing financial interests. 
association study (GWAS) implicated the nature of the HLA-viral peptide interaction as the major genetic factor modulating durable control of HIV-1 infection in the absence of antiretroviral therapy ${ }^{6}$. However, the mechanistic basis for the association remains unclear, and it is also unclear why the majority of persons with so-called 'protective HLA alleles' actually develop progressive disease.

A number of studies have attempted to define quantitative and qualitative differences in $\mathrm{CD} 8^{+} \mathrm{T}$ cell responses that may associate with different outcomes in terms of immune control of viremia. Simple quantitative measures have shown little correlation with viral control $^{7,8}$, suggesting that qualitative features of $\mathrm{CD} 8^{+} \mathrm{T}$ cells may modulate efficacy. Factors potentially modulating protective HLA-associated $\mathrm{CD} 8^{+} \mathrm{T}$ cell responses include, among others, polyfunctionality ${ }^{9}$, antigen sensitivity or functional avidity ${ }^{10,11}$, proliferative capacity ${ }^{12}$, loading of lytic granules ${ }^{13}$, ex vivo expression of perforin ${ }^{14}$, specific targeting of conserved regions 15,16 , immunoregulatory mechanisms ${ }^{17-19}$, concurrent responses to multiple epitopes restricted by different HLA alleles ${ }^{20}, \mathrm{CD} 8^{+} \mathrm{T}$ cell-associated mutations that impair viral fitness ${ }^{21,22}$ and immune escape ${ }^{23}$. Numerous studies also suggest that properties of the T cell receptor (TCR)-peptide-MHC interaction may be involved ${ }^{24,25}$. However, the extent to which any of these factors influences the antiviral efficacy of the human immune response, as reflected by in vivo viral load, remains unclear, in part due to lack of direct comparison of epitope-specific $\mathrm{CD} 8^{+} \mathrm{T}$ cell responses in controllers and progressors, sequence diversity within targeted epitopes and immune escape, and the potential confounding effect of targeting of multiple epitopes through diverse HLA alleles.

To address these limitations, we focused on HIV-1-infected persons expressing HLA$\mathrm{B} * 2705$, a situation in which the immune response is largely if not exclusively mediated by targeting of a single Gag epitope (KK10, KRWIILGLNK, aa $263-272)^{23}$. From a large, well pedigreed cohort ${ }^{26}$, we specifically selected five controllers and five progressors expressing HLA-B $* 2705$, for whom the circulating viruses and cellular proviruses all harbored wild-type sequences within the dominant KK10 epitope targeted through this allele at the time of analysis. This allowed for comparative assessment of adaptive $\mathrm{CD} 8^{+} \mathrm{T}$ cell responses in persons in whom the dominant $\mathrm{CD} 8^{+} \mathrm{T}$ cell response is to a single epitope in $\mathrm{Gag}$, in a setting in which the viral load, and by inference the degree of CD8 ${ }^{+} \mathrm{T}$ cell mediated control, and not HLA allele or sequence variation within the targeted viral epitope, were the primary variables. We performed a detailed analysis of the epitope-specific CD8 ${ }^{+}$ $\mathrm{T}$ cell responses and then extended these to include a dominant $\mathrm{B} * 57$-restricted epitope. These data indicate that HLA-B*27- and HLA-B*57-restricted $\mathrm{CD} 8^{+} \mathrm{T}$ cells targeting the same epitopes in elite controllers and progressors are clearly differentiated based on potency and cross-reactivity of TCR recognition of HIV-1 and viral variants, which is in turn related to specific TCR clonotypes that are selected in natural infection.

\section{Results}

\section{Quantitative measures of KK10-specific T cells}

Previous studies have shown that persons expressing HLA-B*2705 generate an immunodominant response to an epitope in Gag p24 termed KK10 (KRWIILGLNK, aa 263-272), targeting of which is critical to long-term control in these persons ${ }^{23,27}$. Although escape from this response leads to accelerated disease progression, variable viral loads and rates of $\mathrm{CD} 4^{+} \mathrm{T}$ cell decline are already observed before escape occurs ${ }^{23}$. Given the importance of the KK10-specific CD8 ${ }^{+} \mathrm{T}$ cell response to disease control, we reasoned that identification of both controllers and progressors with wild-type KK10 sequence would afford the opportunity to define characteristics of effective and ineffective $\mathrm{CD} 8^{+} \mathrm{T}$ cell responses, independent of any confounding effects of immune escape. 
We recruited 5 HIV-1 elite controllers and 5 HIV-1 progressors for detailed studies, all expressing HLA-B*2705 and having autologous virus containing the wild-type KK10 epitope in plasma HIV-1 RNA and cellular HIV-1 DNA (Table 1). These subjects were selected from a larger population including individuals in whom variants within this epitope were present in vivo. Viral loads in controllers were all <50 RNA copies per ml plasma, and ranged as low as 0.2 RNA copies per ml plasma in those for whom more sensitive testing was performed. Progressors ranged from 4,073 to 22,094 RNA copies per ml plasma (Table 1). The dominance of the KK10-specific responses in these subjects was confirmed by fine mapping with HLA-restricted optimal peptides in interferon- $\gamma$ (IFN- $\gamma$ ) ELISPOT assays (data not shown). Strong KK10-specific $\mathrm{CD} 8^{+} \mathrm{T}$ cell responses as defined by tetramer analysis were detected in both the controllers and progressors expressing wild-type KK10 (Fig. 1a). Comparing the controllers with the progressors, there was no significant difference in magnitude of HLA-B $* 27-$ KK10 tetramer staining $(p=0.7531$; Fig. 1b) or IFN- $\gamma$ production ( $p=0.7383$; Fig. 1c) despite substantial differences in plasma viremia between the two groups.

Since TCR is a key structure that defines antigen recognition, we next evaluated whether differences in TCR usage might be associated with differential ability to control viremia, due to TCR clonotypes with heterogeneous antiviral potential. KK10 tetramer positive cells were sorted and subjected to TCR sequencing. Consistent with the findings in other studies ${ }^{28,29}$, there was striking diversity of clonotype recruitment in all KK10-specific $\mathrm{CD} 8^{+} \mathrm{T}$ cell populations, and despite dominance of single clonotypes in each person, we did not observe preferential usage of a particular TCRBV or CDR3 motif among the various KK10-specific CD8 ${ }^{+} \mathrm{T}$ cell clonotypes (Table 1 ). Indeed, of the clonotypes identified, only two clonotypes were the same in two different subjects (CTR22 and CR420: TCRBV27CASSGGRRAF/J1-1, as well as CTR22 and CR540: TCRBV21-CASTNRGSEQY/J2-7), and only one subject (CR420) possessed a TCRBV4-3/J1-3 clonotype similar to that recently reported in persons expressing HLA-B $* 27$ in whom viral loads varied between 1,880 and $202,590^{28,30,31}$.

These data indicate that $\mathrm{KK} 10$-specific $\mathrm{CD} 8^{+} \mathrm{T}$ cells are quantitatively similar but demonstrate marked heterogeneity in TCR usage among persons targeting a genetically identical epitope through a genetically identical HLA allele, in whom we observe marked differences in viral load.

\section{Functional characteristics of KK10-specific T cells}

A number of reports have suggested qualitative features of $\mathrm{CD} 8^{+} \mathrm{T}$ cells that are associated with viral control. One such measure is antigen sensitivity (often termed 'functional avidity') ${ }^{10,11,31}$, and HLA-B*27 is characterized by high sensitivity T cell responses ${ }^{28}$. However, whether antigen sensitivity varies with viral load in persons expressing HLAB*27 and wild-type epitope has not been determined. We next assessed antigen sensitivity in each of these subjects, by examining IFN- $\gamma$ ELISPOT responses at limiting KK10 peptide concentrations. There was no difference in $\mathrm{SD}_{50}$ between controllers and progressors ( $p=$ 0.4678 ; Fig. 2a). These results, in which all responses were detected in the presence of wildtype KK10 and therefore not confounded by potentially cross-reactive responses induced by mutations within the epitope, are consistent with previous reports showing that the majority of clones specific for KK10 are of similar antigen sensitivity ${ }^{10}$.

Polyfunctionality was next examined, including the capacity of ex vivo B*2705 KK10specific $\mathrm{CD} 8^{+} \mathrm{T}$ cells to simultaneously produce the effector cytokines and chemokines IFN- $\gamma$, IL-2, TNF, and MIP-1 $\beta$ and to release cytotoxic factors by monitoring the expression of the degranulation marker CD107a upon KK10 peptide stimulation. Previous population studies have shown that the ability to produce 4 and 5 functions concurrently is 
associated with HIV-1 controllers ${ }^{9}$, and although some epitope-specific responses have been evaluated in this manner $10,28,32$, this has not been examined for HLA-B $* 27$ restricted responses in persons known to express wild-type virus-in whom the inducing antigen is thus the same. When this analysis was done for the KK10 epitope (Fig. 2b), MIP-1 $\beta$ secreting cells dominated the responses in both progressors and controllers, consistent with previous findings ${ }^{9}$. Pairwise comparisons revealed several subsets with significantly different functional profiles between the two groups. For example, cells dually expressing IFN- $\gamma$ and MIP- $1 \beta$ were significantly higher in controllers $(p=0.031)$, whereas cells dually expressing IFN- $\gamma$ and TNF were higher in progressors $(p=0.012)$. However, although cells with greater than three functions were enriched in controllers, this did not reach statistical significance $(p=0.1)$ and these cells made up only a small subset of the total KK10-specific $\mathrm{CD} 8^{+} \mathrm{T}$ cell response.

Previous studies have shown that chronic HIV-1 infection skews maturation of HIV-1specific $\mathrm{CD} 8^{+} \mathrm{T}$ cells towards pre-terminally differentiated cells with poor cytotoxic activity ${ }^{33}$. CD27 and CD45RA staining was used as described previously ${ }^{34}$ to phenotypically distinguish four distinct subpopulations of KK10-specific cells. Comparable proportions of KK10-specific cells with central memory $\left(\mathrm{KK} 10^{+} \mathrm{CD} 27^{+} \mathrm{CD} 45 \mathrm{RA}^{-}\right)$, effector memory $\left(\mathrm{KK} 10^{+} \mathrm{CD} 27^{-} \mathrm{CD} 45 \mathrm{RA}^{-}\right)$, or terminally differentiated effector memory

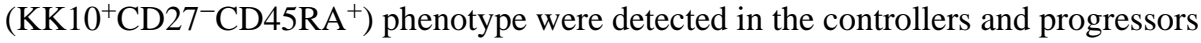
(Supplementary Fig. 1). Moreover, we observed that HIV-1 infected HLA-B*2705-encoding GXR cells stimulated proliferation to a comparable degree in both groups by giving the same signal ( $p=0.2222$; Fig. $2 \mathrm{c}$ and $2 \mathrm{~d})$.

Together, these data indicate that the superior control of wild-type viremia in this cohort of elite controllers and chronic progressors expressing HLA-B*27, all of whom were treatment naive, is not reflected in quantitative measures of KK10-specific CD8 ${ }^{+} \mathrm{T}$ cells, nor in qualitative assessment of their functional avidity, cytokine secretion, proliferative capacity, or differentiation phenotypes.

\section{Virus neutralization by KK10-specific T cells}

Having shown that the above measures of responses to the immunodominant KK10 Gag epitope did not differentiate these controllers from progressors, we next evaluated the functional ability of responses to this epitope to inhibit HIV-1 replication in vitro ${ }^{35}$. We limited the initial analysis to the elite controllers, in whom outgrowth of autologous virus in $\mathrm{CD}^{+} \mathrm{T}$ cells is markedly delayed ${ }^{36}$, allowing us to use controlled inocula of exogenous HIV-1 isolates to infect these cells and measure the ability of defined numbers of CD8 ${ }^{+} \mathrm{T}$ cells to inhibit virus replication. We included all 5 HLA-B*2705-positive elite controllers demonstrated to harbor wild-type KK-10 epitope sequences in plasma virus and PBMC provirus (Table 1) and assessed antiviral ability of bulk $\mathrm{CD} 8^{+} \mathrm{T}$ cells and KK10-specific cell-depleted $\mathrm{CD} 8^{+} \mathrm{T}$ cells to inhibit virus replication in autologous $\mathrm{CD} 4^{+} \mathrm{T}$ cells by measurement of p24 antigen production in the supernatant over 7 days ${ }^{35}$. Addition of bulk $\mathrm{CD}^{+} \mathrm{T}$ cells to virally infected $\mathrm{CD} 4^{+} \mathrm{T}$ cells resulted in a 3 to $4 \log$ reduction in $\mathrm{p} 24$ antigen production at day 7 in culture, whereas viral inhibition was reduced by greater than 90\% using cells in which KK10-specific $\mathrm{CD}^{+} \mathrm{T}$ cells were depleted (Fig. 3a). This confirmed that the major immune control was mediated by the KK10 response in each of these subjects, and showed that all of the $\mathrm{B} * 27$-positive controllers were able to limit virus replication in vitro.

We next sought to examine the antiviral function of the $\mathrm{CD} 8^{+} \mathrm{T}$ cells from the progressors. However, outgrowth of autologous virus in HIV-1 progressors complicates the viral inhibition assay using autologous $\mathrm{CD} 4^{+} \mathrm{T}$ cells (data not shown). We therefore sought to validate an assay based on the use of HLA-B*2705-encoding GFP reporter CEM-derived 
GXR cells as target cells, which fluoresce green upon infection ${ }^{37,38}$. When these target cells were used with bulk $\mathrm{CD} 8^{+} \mathrm{T}$ cells from the controllers, there was marked inhibition of replication; moreover, there was near complete loss of virus inhibition by depletion of KK10-specific cells from bulk CD8 ${ }^{+} \mathrm{T}$ cell population (Fig. 3b). In contrast, no inhibition was observed by bulk $\mathrm{CD} 8^{+} \mathrm{T}$ cells from HIV-1 negative individuals either using virally infected antologous $\mathrm{CD} 4{ }^{+} \mathrm{T}$ cells or HLA-B $* 2705$-encoding GFP reporter GXR cells. In addition, no inhibition was observed by bulk $\mathrm{CD} 8^{+} \mathrm{T}$ cells from the controllers using HLAB*2705-negative GFP reporter GXR cells after infection of the same virus (data not shown).

Having shown that this assay provides evidence of active virus neutralization by ex vivo KK10-specific CD8 ${ }^{+} \mathrm{T}$ cells in HLA-B*2705-positive elite controllers, and that this assay is sensitive to KK10 epitope specificity and HLA-B*2705 expression, we next evaluated CD8 ${ }^{+}$ T cells from the HIV-1 progressors. Infected GXR cells expressing HLA-B*2705 were inhibited by addition of $\mathrm{CD}^{+} \mathrm{T}$ cells from progressors (Fig. 3c), but to a dramatically lesser degree than had been seen with the controllers $(p<0.0001)$. This was observed despite the fact that there were no quantitative differences in KK10-specific cell numbers comparing the controllers to progressors, as shown by tetramer staining and IFN- $\gamma$ ELISPOT assay.

We next extended these studies to determine the ability of KK10-specific $\mathrm{CD} 8^{+} \mathrm{T}$ cell responses in controllers and progressors to recognize viral escape variants known to arise in vivo ${ }^{38}$. Recent computational studies suggest that protective HLA alleles are associated with enhanced cross-reactivity ${ }^{25}$, but this has not been evaluated in the context of a single HLA class I allele and single epitope, comparing controllers and progressors. Recognition of HIV-1 and viral variants by KK10-specific $\mathrm{CD}^{+} \mathrm{T}$ cells in bulk $\mathrm{CD} 8^{+} \mathrm{T}$ cells from the controllers and progressors was analyzed by flow cytometry. This was done by evaluating the proportion of GFP-positive cells after infecting HLA-B*2705-encoding GFP reporter GXR cells. We chose the GFP reporter GXR cell assay system rather than autologous CD4 ${ }^{+}$ T cells, again to overcome outgrowth of autologous virus from the chronic progressors and avoid potential variability of $\mathrm{CD} 4^{+} \mathrm{T}$ cell responses among study subjects.

An example of recognition of HIV-1 NL4-3 harboring KK10 epitope (KRWIILGLNK) and KK10 viral variants (Table 2) by KK10-specific CD8 ${ }^{+} \mathrm{T}$ cells from an HLA-B*2705positive elite controller (FW56) and a chronic progressor (CR540) is shown in Fig. 4a. There was essentially complete inhibition of wild-type NL4-3 virus replication and broad recognition of viral variants by KK10-specific $\mathrm{CD}^{+} \mathrm{T}$ cells from controller FW56, as well as marked inhibition of the typical early L6M mutant, which was not detected in this individual. In contrast, inhibition of both of these targets by $\mathrm{CD} 8^{+} \mathrm{T}$ cells from progressor CR540 was present but minimal. Although peak infection with the other mutant viruses was less than with wild-type virus or L6M mutant, controller FW56 inhibited all variants, whereas $\mathrm{CD}^{+} \mathrm{T}$ cells from progressor CR540 were ineffective, even though both subjects had comparable KK10-specific effector cell proportions quantified by tetramer staining (Table 1) and proliferative capacity in response to HIV-1 infected HLA-B*2705-encoding GXR cells (Fig. 2c). The superiority in antiviral efficacy against NL4-3 wild-type virus and viral variants by HIV-1-specific $\mathrm{CD}^{+} \mathrm{T}$ cells from elite controllers was consistently observed when these detailed studies were extended to the 5 elite controllers and 5 chronic progressors ( $p<0.0001$; Fig. $4 \mathrm{~b})$. In addition, bulk CD ${ }^{+} \mathrm{T}$ cells from HIV-1 negative individuals ( $\mathrm{n}=12$, including 4 HLA-B*2705-positive donors) did not inhibit virus replication in B*2705-encoding GFP reporter GXR cells (Fig. 4b). Similar results in terms of killing efficacy against the same virus-infected HLA-B*2705-encoding GXR cells were observed when we performed standard chromium release assays in the controllers and progressors ( $p<0.0001$; Fig. 4c). 
Together, these data indicate that HLA-B*2705-restricted CD $8^{+} \mathrm{T}$ cells targeting the KK10 epitope can be clearly differentiated between the elite controllers and chronic progressors based on potency and cross-reactivity of recognition of cells infected with wild-type HIV-1 and HIV-1 containing naturally arising mutations in the KK10 epitope. These cross-reactive neutralization data are consistent with the observation that the CDR3 sequences of KK10specific clonotypes were significantly closer to germline in the controllers compared to the progressors ( $p=0.001$; Supplementary Fig. 2 ), which confers a greater ability to recognize epitope variants ${ }^{39}$. These functional data are also consistent with computational modeling showing that thymic selection in the context of protective HLA alleles is more likely to generate a cross-reactive $\mathrm{CD} 8^{+} \mathrm{T}$ cell repertoire targeting mutants of viral epitopes, thereby contributing to improved control of a highly variable pathogen 25 .

\section{Antiviral efficacy of KK10-specific clonotypes}

The above data indicate that there are differences in potency and cross-reactivity of recognition of wild-type HIV-1 and viral variants in the KK10-specific CD8 ${ }^{+} \mathrm{T}$ cell responses between controllers and progressors, suggesting that the fine specificity of the TCR might be modulating these effects. Given that we had observed different clonotypes in the tetramer-positive populations in these ten subjects (Table 1), we next sought to determine clonotypic antiviral efficacy. We therefore cloned HLA-B*2705 KK10-specific $\mathrm{CD} 8^{+} \mathrm{T}$ cells by limiting dilution from the KK10 tetramer-sorted cells from three elite controllers (CTR203, FW56 and CTR40) and two chronic progressors (CR540 and CR420), and then determined clonotypes by TCR sequencing (Table 3 ). In each of these individuals, the dominant clonotypes identified in vivo was cloned, and in 3 of the five subjects we were able to generate multiple clones. In addition, for multiple subjects we were able to establish clones for additional subdominant clonotypes.

We tested the ability of the clonotypic KK10-specific CD8 ${ }^{+} \mathrm{T}$ cells to kill virally infected target cells in a standard chromium release assay, and to inhibit virus replication, again using the HLA-B*2705-encoding GFP reporter GXR cells. For controller CTR203, we were able to establish clones representing 5 of the 6 clonotypes detected in peripheral blood. We observed marked variation in the ability of these clonotypes to recognize HIV-1 and viral variants (Fig. 5), ranging from broad recognition of the variants by the two most dominant CTR203 clonotypes, to weak and narrow recognition by all three CTR203 subdominant TCR variants. Extending this to clones from five subjects, the most effective clonotypes were the immunodominant in vivo clonotypes from the controllers, including the two codominant responses in subject CTR203, the two co-dominant responses in subject FW56 and the one dominant response in subject CTR40. These were significantly more potent at viral recognition than the immunodominant clones from the progressors $(p=0.005)$. Co-dominant clonotype TCRBV4-3 in controller FW56, which exhibited efficient recognition of wildtype virus and had less robust activity against the L6M variant and was unable to recognize any of the other variants, was observed at a low frequency in progressors CR540, CR420 and 8222 in the context of different CDR3 or J segments.

Subdominant clonotypes from the elite controllers included TCRBV27/J2-2, TCRBV20-1/ J2-7 and TCRBV20-1/J1-2, all of which were associated with inferior recognition of wildtype virus and the L6M variant and exhibited the least efficacy against the other viral variants. However, these less effective clonotypes were dominantly selected by progressors CR338, 8222 and CR420. They were rearranged with variant CDR3 or J segments, but were likewise less efficient at recognizing virally infected cells. And none of the subdominant clonotypes, whether from controllers or progressors, was able to efficiently recognize virally infected cells or to inhibit virus replication. Consistent findings in terms of the ability of individual clonotypes to inhibit virus replication were observed (Supplementary Fig. 3). 
Together, these data indicate that differential antiviral efficacy of KK10-specific CD8 ${ }^{+} \mathrm{T}$ cells in HIV-1-infected persons is defined by dominance of clonotypes which confer distinct antiviral potential on $\mathrm{CD} 8^{+} \mathrm{T}$ cells.

\section{Antiviral efficacy of $B^{\star} 57-T W 10$ specific clonotypes}

The effect of individual TCR clonotypes on antiviral efficacy was further examined with HLA-B*5701-restricted TW10 (TSTLQEQIGW, Gag residues 240 to 249)-specific CD8 ${ }^{+}$T cell clones in the chromium assay with virally infected HLA-B*5701-encoding GFP reporter GXR cells (Fig. 6). HLA-B*5701-restricted TW10-specific CD8 ${ }^{+} \mathrm{T}$ cell clones were generated by limiting dilution from TW10 (TSTLQEQIGW) tetramer-sorted cells from HLA-B*57-positive elite controllers (CTR53, CR462) and a chronic progressor (CR555) and then clonotypically determined by TCR sequencing. Again, we observed that variant clonotypes possessed differential antiviral efficacy and that overall clones from the elite controllers were more potent and cross-reactive in recognition of HIV-1 and viral variants than clones from the progressor.

Together, these data indicate that the difference of the same epitope-specific CD8 ${ }^{+} \mathrm{T}$ cell responses between the controllers and progressors in recognition of HIV-1 and viral variants is related to distinct TCR clonotypes that are selected in natural infection.

\section{Lytic granule loading and delivery by clonotypes}

The above data indicate clonotype-specific functional differences in antiviral function, offering the opportunity to define mechanisms that account for these phenotypes. We next determined the effect of TCR clonotype on lytic granule loading upon recognition of HIVinfected target cells, and the ability of these $\mathrm{T}$ cells to deliver perforin to infected target cells. Expression of perforin and granzyme $\mathrm{B}(\mathrm{GrB})$ by representative clonotypes was measured by flow cytometry ${ }^{12,13}$. Upon culture with KK10 wild-type HIV-1-infected HLAB*2705-expressing GXR cells for 3 days, dominant clonotypes from controllers efficiently expressed perforin. In contrast, subdominant clonotypes from controllers and all the clonotypes from progressors were significantly less efficient at expressing perforin $(p<$ 0.0001 ; Fig. 7a). Similar results were observed for GrB expression $(p<0.0001$; Supplementary Fig. 4).

We also examined effector-target cell conjugation and granule loading and delivery by confocal microscopy ${ }^{40}$. Upon incubation with virally infected HLA-B*2705-expressing GXR cells for 30 minutes, more perforin in the inhibitory clonotypes was polarized to synapses and released into target cells, as compared to the non-inhibitory clonotypes (Fig. $7 b)$. An extended quantitative analysis of perforin loading and delivery showed that significantly less perforin was delivered to synapses $(p<0.0001$; Fig. $7 \mathrm{c})$ and released into target cells $(p<0.0001 ;$ Fig. $7 \mathrm{~d})$ by subdominant clonotypes from controllers and all the clonotypes from progressors. As control, endogenous perforin was confirmed to be undetectable in virally infected GXR cells (Supplementary Fig. 5). Comparable basal levels of perforin were observed in inhibitory and non-inhibitory clonotypes without target cell stimulation ( $p=0.96$; Supplementary Fig. 6 ), indicating that the functionally effective clones rapidly upregulate perforin loading upon cognate antigen recognition.

These data indicate that TCR clonotypes that are associated with enhanced abillty to inhibit HIV-1 replication do so by rapid upregulation of lytic granules at the immunological synapes following target cell engagement, and delivery of these into the infected cell. 


\section{Discussion}

Certain HLA B alleles are associated with enhanced control of viremia in HIV-1 infected persons ${ }^{3-5}$, and this effect maps to specific host amino acids within the HLA-B peptide binding groove ${ }^{6}$. However, the majority of persons with so-called protective alleles such as HLA B*27 and HLA-B*57 experience progressive infection ${ }^{26}$. To address the basis for these differences in outcome, we compared $\mathrm{CD}^{+} \mathrm{T}$ cell responses to immunodominant epitopes in treatment-naive elite controllers and chronic progressors. In order to limit the number of potential confounding variables, all subjects harbored wild-type sequences of the respective $T$ cell epitopes in plasma virus and PBMC provirus, and all shared the same HLA class I restricting allele. In this setting, in which contemporaneous immune escape is not a confounding issue, we find that $\mathrm{CD} 8^{+} \mathrm{T}$ cell responses from HIV-1 controllers are more potent at inhibiting HIV-1 replication than those in progressors targeting the same epitopes, and better able to cross-recognize HIV-1 viral variants that typically arise in vivo. Moreover, these effects are associated with a unique ability of the dominant TCR clonotypes to upregulate perforin and $\mathrm{GrB}$, providing a mechanistic explanation for the divergent disease outcomes in persons with protective HLA alleles.

There have been numerous characteristics of $\mathrm{CD} 8^{+} \mathrm{T}$ cells that have been reported to be associated with enhanced control of viremia, including differences in polyfunctionality, proliferative capacity, and functional avidity of KK10-specific $\mathrm{CD} 8^{+} \mathrm{T}$ cells. In the carefully controlled comparative studies done here, in a small cohort of well pedigreed controllers and progresssors expressing protective alleles, none of these previously reported associations reached statistical significance. In contrast, functional ability of both bulk $\mathrm{CD} 8^{+} \mathrm{T}$ cells as well as epitope-specific TCR clonotypes to inhibit virus replication, cross recognize viral vaiants and upregulate perforin and $\mathrm{GrB}$, which are likely the most important in vivo function of these cells, were highly significant. Overall, this study links the antiviral efficacy of the two most protective HLA class I alleles to CD8 ${ }^{+} \mathrm{T}$ cell clonotypes selected in HIV-1 natural infection to viral control in vivo, and demonstrates that TCR rearrangement modulates the effect of protective alleles on disease outcome.

This study is distinct from other reports of TCR clonotype usage by KK10-specific CD8 ${ }^{+} \mathrm{T}$ cells in persons expressing HLA-B*27, in that the subjects here were stratified by extremes of viral load. As in previous studies ${ }^{28,29}$, we observed striking diversity of clonotype recruitment and CDR3 motif in KK10-specific $\mathrm{CD}^{+} \mathrm{T}$ cell populations, as well as a dominance of clonotypes in progressors that were unable to cross-recognize the L6M mutation. This mutation is known to occur early during the course of HIV-1 infection with little or no impact on peptide processing ${ }^{27}$, HLA-B $* 27$ binding ${ }^{27}$, TCR recognition ${ }^{41}$, or viral fitness ${ }^{38}$, but it is an important intermediate mutation on the path to complete escape. Although such ineffective responses were detected at a clonal level in both controllers and progressors in our cohort, HLA-B*27 positive elite controllers possess dominant clonotypes that not only target the L6M mutant, but also other mutants including substitution at KK10 residue position 2 alone or in combination with the L6M mutation, which reduce peptide binding to HLA-B*27 and impair viral replication ${ }^{38}$. Thus the TCR clonotypes in controllers are comprised of effective and ineffective clonotypes, whereas those in progressors are limited to less effective clonotypes.

In contrast to some other reports ${ }^{42,43}$, we found no significant differences in the usage of public clonotypes between the two groups despite siginificant differences in plasma viremia and that public clonotypes do not appear to dominate among controllers ${ }^{29}$. However, controllers used TCRB clonotypes with CDR3 sequences that were significantly more "germline-like" than those used by the progressors. The lower number of nucleotide additions in the germline-like CDR3 regions is a hallmark of clonotypes that are found at 
high frequecny in naive and memory $\mathrm{T}$ cell pools and that are also shared between multiple individuals ${ }^{44}$. The mechanism underlying this advantage bestowed by germline-like CDR3 regions may be related to higher precursor frequency and (or) greater ability to recognize mutational variants of the epitope ${ }^{39,42}$. Furthermore, the most effective clonotypes, in terms of viral inhibition and cytotoxic recognition of wild-type and variant viruses, were dominantly selected in vivo by the controllers but either absent or subdominantly selected in KK10-specific $\mathrm{CD}^{+} \mathrm{T}$ cell populations by the progressors. In contrast, the clonotypes associated with inferior recognition of wild-type virus and the least efficacy against the viral variants were dominantly selected by the progressors but subdominantly selected by the controllers.

Although this study clearly shows that TCR modulates the protective effect of HLA alleles, it has a number of limitations. The HLA-B*2705 studies were limited to only 5 controllers and 5 progressors, and a number of previously reported associations with viral control did not reach statistical significance in this small study group. Nevertheless, even with these small numbers the results are highly significant in showing greater cytotoxic killing and greater cross-reactivity by the dominant clonotypes in controllers compared to progressors. We were not able to generate $\mathrm{CD} 8^{+} \mathrm{T}$ cell clones representing all detectable TCR clonotypes in all persons, but in one subject we were able to test 5 of 6 clonotypes in vivo, representing $90 \%$ of the detectable TCR diversity in that subject. Moreover, all three controllers evaluated at the clonal level had dominant TCR clonotypes that were highly effective, whereas these were absent in both dominant and subdominant clones established in the progressors. Of note, the important role of TCR clonotypes in distinguishing virus control from lack of control likely was mediated by direct cytotoxicity of HIV-1-infected cells, with perforin upregulation noted within 30 minutes of cognate epitope recogntion and not requiring proliferation of $\mathrm{CD} 8^{+} \mathrm{T}$ cells. The data presented here contrast with the well documented differential proliferative capacity of $\mathrm{CD} 8^{+} \mathrm{T}$ cells between nonprogressors and progressors ${ }^{12}$, likely due to the way in which proliferation was measured. In the current study, we used virally infected GXR cells expressing HLA-B*2705 as stimulator cells in culture with bulk $\mathrm{CD} 8^{+} \mathrm{T}$ cells and observed comparable proliferative capacity of HLA$\mathrm{B} * 27 \mathrm{KK} 10$-specific $\mathrm{CD} 8^{+} \mathrm{T}$ cells between the controllers and progressors. This could be explained by the comparable level of $\mathrm{CD}^{+} \mathrm{T}$ cells $(p=0.4206)$ in the progrssors and controllers, properties of $\mathrm{T}$ regulatory cells in persons expressing protective HLA alleles ${ }^{17}$, or cytokines produced by the cell line used for stimulation compensating for in vivo impaired $\mathrm{CD}^{+} \mathrm{T}$ cell helper function in progressors. Finally, whether these results can be extrapolated to other protective alleles and other epitopes will require additional study.

Taken together, our data indicate that TCR usage modulates viral inhibitory capacity and recognition of naturally arising HIV-1 variants, and thus modulates the effect of protective HLA alleles. The data suggest that TCR clonotypes that inhibit viral replication and confer cross-recognition of viral epitope variants that can eventually arise in vivo may be critical to long term control of viremia. Efforts to define the factors that contribute to junctional rearrangement of more effective TCR may be of critical importance for T cell vaccine design and therapeutic strategies for highly variable pathogens like HIV-1.

\section{Materials and Methods}

\section{Study subjects}

PBMC and plasma samples from HIV-1-infected individuals and HIV-1 negative persons were used for this study according to protocols approved by the Institutional Review Board of the Massachusetts General Hospital. Elite controllers were defined as having HIV-1 RNA below the level of detection for the respective available ultrasensitive assay (e.g., < 75 RNA copies/ml by bDNA or $<50$ copies by ultrasenstive PCR) without antiretroviral therapy. 
Treatment-naive chronic progressors in the study had a median virus load of 12,833 copies/ $\mathrm{ml}$ (4073 - 22,094 copies/ml). CD4 ${ }^{+} \mathrm{T}$ cell counts, viral loads and HLA types were determined as described ${ }^{26}$. Characteristics of the study subjects are shown in Table 1.

\section{Viruses and synthetic peptides}

The CXCR4-utilizing HIV-1 laboratory strain NL4-3 was obtained from the AIDS Research and Reference Reagent Program, Division of AIDS, NIAID, NIH (Bethesda, Maryland, USA). HIV-1 laboratory strain NL4-3 was also modified to express one or more mutations in Gag p24 as previously described ${ }^{38,45}$. Peptides corresponding to described optimal HIV-1 epitopes and their variants (http://hiv-web.lanl.gov) were synthesized at the MGH Peptide Core Facility on an automated peptide synthesizer using F-moc technology.

\section{Virus sequencing}

Nested PCR for viral DNA or RNA was performed as previously described ${ }^{46}$. PCR fragments were population sequenced to identify regions of sequence variation. All fragments were sequenced bi-directionally on an ABI 3730×1 automated sequencer (Applied Biosystems, Foster City, CA).

\section{ELISPOT assay}

IFN- $\gamma$ enzyme-linked immunospot (ELISPOT) assays were performed as described, using optimally defined epitopes and designated concentrations of peptide ${ }^{8}$. Input cells ranged from 10,000 to 100,000 per well. To calculate the number of specific spot-forming cells (SFC), the number of spots in the negative control wells was subtracted from the number of spots in each experimental well. Responses were regarded as positive if they had at least 3 times the mean number of SFC in the 3 negative control wells; positive responses also had to be at least $50 \mathrm{SFC} / 10^{6} \mathrm{PBMCs}$. The magnitude of epitope-specific response was calculated as SFC per million cells.

\section{Generation of $\mathrm{CD}^{+} \mathrm{T}$ cell clones}

PBMC were stained with fluorophore-labeled HLA tetramer refolded with epitopic HIV-1 peptides (ProImmune, Oxford, UK) and fluorophore-labeled anti-CD8 and anti-CD3 antibodies. Tetramer-positive, CD8-positive cells were sorted on a FACS Aria cell-sorting instrument (BD Biosciences) at 70 pounds per square inch (PSI) and single cells were placed into each well of 96-well plates, using irradiated allogeneic PBMC and CD3-specific $\mathrm{mAb}$ $12 \mathrm{~F} 6$ as a stimulus for $\mathrm{T}$ cell proliferation ${ }^{47}$. Developing epitope-specific clones were further tested in IFN- $\gamma$ ELISPOT assays with optimal epitopes and with tetramer staining. Cloned $\mathrm{CD} 8^{+} \mathrm{T}$ cells were maintained by restimulation every 14 to 21 days with an anti$\mathrm{CD} 3 \mathrm{mAb}$ and irradiated allogeneic PBMC in RPMI 1640 medium containing $50 \mathrm{U} / \mathrm{ml}$ of recombinant IL-2, as described ${ }^{47}$.

\section{TCR $\alpha$ and $\beta$ chain sequencing}

Tetramer-positive CD8-positive cells were sorted from PBMC or cloned $\mathrm{CD} 8^{+} \mathrm{T}$ cells and mRNA was extracted using the RNeasy mini kit (Qiagen, Valencia, CA). Anchored RTPCR was then performed using a modified version of the SMART (switching mechanism at $5^{\prime}$ end of RNA transcript) procedure and a TCR $a$ or $\beta$ chain constant region $3^{\prime}$-primer to obtain PCR products containing the $\mathrm{Va}$ or $\mathrm{V} \beta$ chain in addition to the CDR3 region, the Ja/ $\beta$ region and the beginning of the $\mathrm{Ca} / \beta$ region. RT-PCR and TCR $a$ and $\beta$ chain gene sequencing and analysis were performed as described previously ${ }^{48}$. 


\section{Flow cytometry}

Cell staining was performed as previously described ${ }^{9}$. Briefly, cells were stained with indicated tetramer (ProImmune, Oxford, UK) for 20 minutes at room temperature.

Following one wash with PBS containing 1\% FCS, the cells were stained with surface antibodies. After 30 minutes at room temperature, the cells were washed and fixed using the Cytofix/Cytoperm kit (BD PharMingen) according to the manufacturer's instructions. Following fixation, the cells were washed twice in the perm wash buffer and stained with antibodies against intracellular markers. Following staining, the cells were re-suspended in PBS containing 2\% paraformaldehyde. The cells were acquired on a LSRII cytometer (BD Biosciences). Flow data were analyzed with the FlowJo software package (Treestar, Ashland, OR).

\section{Proliferation assay}

Primary $\mathrm{CD} 8^{+} \mathrm{T}$ cells were isolated from PBMC by negative selection (Dynabeads, Invitrogen) with the proportion of $\mathrm{CD}^{+} \mathrm{CD}^{+} \mathrm{T}$ cells $>98 \%$ detected by flow cytometry. Cells were stained with $0.35 \mu \mathrm{M}$ carboxyfluorescein diacetate succinimidyl ester (CFSE; Molecular Probes, Breda, Netherlands) for $7 \mathrm{~min}$ at $37^{\circ} \mathrm{C}$ and then cultured with medium alone or with HIV-1 infected or uninfected HLA-B*27-encoding CEM-derived GXR cells for 7 days in RPMI 1640 medium in the absence of IL-2. After labeling with indicated tetramer (ProImmune, Oxford, UK), anti-CD8 and anti-CD3 antibodies, cells were fixed in $1 \%$ paraformaldehyde and analyzed on an LSRII flow cytometer (BD Biosciences).

\section{Chromium release assay}

HLA-B*27- or HLA-B*57-expressing green fluorescent protein (GFP) reporter CEMderived GXR cells were constructed as described elsewhere 37,38 and infected with the designated HIV-1 strains or viral variants at the specified multiplicity of infection (MOI). On day 5 after infection, viable virally infected cells (which contain a plasmid encoding GFP driven by the HIV-1 long terminal repeat) were sorted on a FACS Aria cell-sorting instrument (BD Biosciences) and labeled with chromium for $1 \mathrm{~h}$ at $37^{\circ} \mathrm{C}$. Bulk CD8 $8^{+} \mathrm{T}$ cells isolated from PBMC by negative selection (Dynabeads, Invitrogen) or $\mathrm{CD} 8^{+} \mathrm{T}$ cell clones were then added at the indicated effector-target ratios, and a standard 4-h chromium release assay was performed as previously described ${ }^{49}$. Percent specific lysis was calculated as $[($ mean experimental $\mathrm{cpm}$ - mean spontaneous $\mathrm{cpm}) /($ mean maximum $\mathrm{cpm}$ - mean spontaneous $\mathrm{cpm})] \times 100$. Spontaneous and maximum releases were determined by incubating the labeled target cells with medium alone or $2 \%$ Triton X-100, respectively.

\section{Viral inhibition assay}

HLA-B*27- or HLA-B*57-expressing GFP reporter CEM-derived GXR cells were infected with the designated HIV-1 strains and viral variants at the specified MOI for $4 \mathrm{~h}$ at $37^{\circ} \mathrm{C}$, then washed and cocultured with bulk $\mathrm{CD} 8^{+} \mathrm{T}$ cells isolated from PBMC by negative selection (Dynabeads, Invitrogen) or $\mathrm{CD} 8^{+} \mathrm{T}$ cell clones at the indicated effector-target cell ratios. The ability to recognize HIV-1 and viral variants by $\mathrm{CD} 8^{+} \mathrm{T}$ cells was analyzed by flow cytometry to evaluate the proportion of GFP-positive cells over 7 days in culture. To additionally address the relative antiviral efficacy of epitope-specific $\mathrm{CD} 8^{+} \mathrm{T}$ cell responses we measured the ability of bulk CD8 ${ }^{+} \mathrm{T}$ cells and epitope-specific cell-depleted $\mathrm{CD} 8^{+} \mathrm{T}$ cells to inhibit virus replication in autologous primary $\mathrm{CD} 4^{+} \mathrm{T}$ cells by analysis of $\mathrm{p} 24$ production, as described elsewhere ${ }^{35}$. Briefly, primary $\mathrm{CD} 4^{+} \mathrm{T}$ cells were isolated from PBMC by negative selection (Dynabeads, Invitrogen). Greater than $98 \%$ of these primary cells coexpressed CD3 and CD4 by flow cytometric analysis. These $\mathrm{CD} 4{ }^{+} \mathrm{T}$ cells were stimulated with CD3-CD8-bispecific monoclonal antibody ${ }^{50}$ and infected at day 3 with the designated HIV-1 isolates at a MOI of 0.1, except as otherwise specified, for $4 \mathrm{~h}$ at $37^{\circ} \mathrm{C}$. 
Virally infected cells were then washed and incubated in the absence or presence of effector cells at an effector-to-target cell ratio of 1:1 in RPMI 1640 medium in addition of IL-2 at 50 $\mathrm{U} / \mathrm{ml}$. At regular intervals, the cultures were fed by removing and replacing one-half of the culture supernatant with fresh medium. The removed supernatant was cryopreserved for subsequent p24 antigen quantitation by ELISA (Dupont, Boston, MA). Virus inhibition was calculated as follows: $\%$ inhibition $=100 \times\left[1-\left(\left[\% \mathrm{GFP}^{+}\right.\right.\right.$cells with effectors $] /\left[\% \mathrm{GFP}^{+}\right.$ cells without effectors])].

\section{Effector-target cell conjugation, granule loading and delivery}

$\mathrm{CD}^{+} \mathrm{T}$ cell clones were cultured with HIV-1 infected or uninfected HLA-B*27-expressing GFP reporter GXR cells for 30 minutes in RPMI 1640 medium in the absence of IL-2. Perforin staining in $\mathrm{CD}^{+} \mathrm{T}$ cell clones was as described ${ }^{40}$. Briefly, cells were fixed with freshly prepared $4 \%$ paraformaldehyde for 15-30 minutes at room temperature and washed with PBS for 3 times. Cells were permeabilized in 0.5\% Triton-X100 (Sigma) and 10\% normal donkey serum (NDS; Jackson Immunoresearch, West Grove, PA) in PBS for 30 minutes at room temperature. Cells were stained with anti perforin monoclonal antibody mouse IgG2b for 60 minutes at room temperature. The primary antibody was diluted with $0.05 \%$ Triton-X100 and 3\% NDS in PBS (1:333 dilution). After three washes in PBS, cells were incubated for 1 hour at room temperature with appropriate secondary antibodies in $0.05 \%$ Triton-X100 and 3\% NDS in PBS. All secondary antibodies used were Alexa Fluor dyes conjugated (1:1000 dilution). F-actin was stained with phalloidin-Alexa Fluor 647 (1:50 dilution). Confocal images were collected on a Zeiss LSM510 Meta Confocal microscope using a plan apochromat $63 \times 1.4$ oil immersion objective. Differential interference contrast (DIC) images were collected simultaneously with the fluorescent images. Multi-track acquisition mode was used to avoid crosstalk between the different fluorophores. Images were analyzed with Imaris software (Bitplane).

\section{Statistical analyses}

An unpaired $t$-test with Welch's correction and Mann-Whitney tests were performed using GraphPad Prism version 4.0a. All tests were two-tailed and $p$-values of $p<0.05$ were considered significant.

\section{Supplementary Material}

Refer to Web version on PubMed Central for supplementary material.

\section{Acknowledgments}

We thank all study participants for their contributions. This work was supported by the Harvard University Center for AIDS Research (5 P30 AI060354-04), grants from the Bill and Melinda Gates Foundation (B.D.W and D.C.D.), the Doris Duke Charitable Foundation (B.D.W.), the NIH (B.D.W. AI030914 and T.M.A. AI074415), the Howard Hughes Medical Institute (B.D.W.), the Mark and Lisa Schwartz Foundation (B.D.W.), the Intramural Research Program and the Office of AIDS Research of the NIH (D.C.D. and S.D.), the New Investigator Award from the Canadian Institutes for Health Research (Z.L.B.), and the Canada Research Chair in Viral Pathogenesis and Immunity (M.A.B.).

\section{References}

1. Migueles SA, Connors M. Long-term Nonprogressive Disease Among Untreated HIV-Infected Individuals. JAMA: The Journal of the American Medical Association. 2010; 304:194-201. [PubMed: 20628133]

2. Deeks SG, Walker BD. Human Immunodeficiency Virus Controllers: Mechanisms of Durable Virus Control in the Absence of Antiretroviral Therapy. Immunity. 2007; 27:406-416. [PubMed: 17892849] 
3. Kaslow RA, et al. Influence of combinations of human major histocompatibility complex genes on the course of HIV-1 infection. Nat Med. 1996; 2:405-411. [PubMed: 8597949]

4. Migueles SA, et al. HLA B*5701 is highly associated with restriction of virus replication in a subgroup of HIV-infected long term nonprogressors. PNAS. 2000; 97:2709-2714. [PubMed: 10694578]

5. Carrington M, O'Brien SJ. The Influence of HLA Genotype on AIDS*. Annual Review of Medicine. 2003; 54:535-551.

6. Pereyra F, et al. The major genetic determinants of HIV-1 control affect HLA class I peptide presentation. Science. 2010; 330:1551-7. [PubMed: 21051598]

7. Betts MR, et al. Analysis of total human immunodeficiency virus (HIV)-specific CD4 ${ }^{+}$and $\mathrm{CD}^{+}$ T-cell responses: relationship to viral load in untreated HIV infection. J Virol. 2001; 75:11983-91. [PubMed: 11711588]

8. Addo MM, et al. Comprehensive epitope analysis of human immunodeficiency virus type 1 (HIV-1)-specific T-cell responses directed against the entire expressed HIV-1 genome demonstrate broadly directed responses, but no correlation to viral load. J Virol. 2003; 77:2081-92. [PubMed: 12525643]

9. Betts MR, et al. HIV nonprogressors preferentially maintain highly functional HIV-specific $\mathrm{CD}^{+} \mathrm{T}$ cells. Blood. 2006; 107:4781-9. [PubMed: 16467198]

10. Almeida JR, et al. Antigen sensitivity is a major determinant of $\mathrm{CD} 8^{+} \mathrm{T}$-cell polyfunctionality and HIV-suppressive activity. Blood. 2009; 113:6351-60. [PubMed: 19389882]

11. Bennett MS, Ng HL, Dagarag M, Ali A, Yang OO. Epitope-Dependent Avidity Thresholds for Cytotoxic T-Lymphocyte Clearance of Virus-Infected Cells. J. Virol. 2007; 81:4973-4980. [PubMed: 17329324]

12. Migueles SA, et al. HIV-specific $\mathrm{CD}^{+} \mathrm{T}$ cell proliferation is coupled to perforin expression and is maintained in nonprogressors. Nat Immunol. 2002; 3:1061-8. [PubMed: 12368910]

13. Migueles SA, et al. Lytic Granule Loading of $\mathrm{CD}^{+}{ }^{+} \mathrm{T}$ Cells Is Required for HIV-Infected Cell Elimination Associated with Immune Control. Immunity. 2008; 29:1009-1021. [PubMed: 19062316]

14. Hersperger AR, et al. Perforin expression directly ex vivo by HIV-specific CD8 T-cells is a correlate of HIV elite control. PLoS Pathog. 2010; 6:e1000917. [PubMed: 20523897]

15. Dahirel V, et al. Coordinate linkage of HIV evolution reveals regions of immunological vulnerability. Proceedings of the National Academy of Sciences. 2011; 108:11530-11535.

16. Kiepiela $\mathrm{P}$, et al. $\mathrm{CD} 8^{+} \mathrm{T}$-cell responses to different HIV proteins have discordant associations with viral load. Nat Med. 2007; 13:46-53. [PubMed: 17173051]

17. Elahi S, et al. Protective HIV-specific $\mathrm{CD}^{+}{ }^{+} \mathrm{T}$ cells evade Treg cell suppression. Nat Med. 2011; 17:989-995. [PubMed: 21765403]

18. Kaufmann DE, et al. Upregulation of CTLA-4 by HIV-specific CD4 ${ }^{+}$T cells correlates with disease progression and defines a reversible immune dysfunction. Nat Immunol advanced online publication. 2007

19. Day CL, et al. PD-1 expression on HIV-specific T cells is associated with T-cell exhaustion and disease progression. Nature. 2006; 443:350-4. [PubMed: 16921384]

20. Perez CL, et al. Broadly Immunogenic HLA Class I Supertype-Restricted Elite CTL Epitopes Recognized in a Diverse Population Infected with Different HIV-1 Subtypes. The Journal of Immunology. 2008; 180:5092-5100. [PubMed: 18354235]

21. Miura T, et al. HLA-B57/B*5801 Human Immunodeficiency Virus Type 1 Elite Controllers Select for Rare Gag Variants Associated with Reduced Viral Replication Capacity and Strong Cytotoxic T-Lymphotye Recognition. J. Virol. 2009; 83:2743-2755. [PubMed: 19116253]

22. Lassen KG, et al. Elite Suppressor Derived HIV-1 Envelope Glycoproteins Exhibit Reduced Entry Efficiency and Kinetics. PLoS Pathog. 2009; 5:e1000377. [PubMed: 19360131]

23. Goulder PJ, et al. Late escape from an immunodominant cytotoxic T-lymphocyte response associated with progression to AIDS. Nat Med. 1997; 3:212-7. [PubMed: 9018241]

24. Lee K-H, et al. The Immunological Synapse Balances T Cell Receptor Signaling and Degradation. Science. 2003; 302:1218-1222. [PubMed: 14512504] 
25. Kosmrlj A, et al. Effects of thymic selection of the T-cell repertoire on HLA class I-associated control of HIV infection. Nature. 2010; 465:350-354. [PubMed: 20445539]

26. Pereyra F, et al. Genetic and Immunologic Heterogeneity among Persons Who Control HIV Infection in the Absence of Therapy. The Journal of Infectious Diseases. 2008; 197:563-571. [PubMed: 18275276]

27. Goulder PJ, et al. Evolution and transmission of stable CTL escape mutations in HIV infection. Nature. 2001; 412:334-8. [PubMed: 11460164]

28. Almeida JR, et al. Superior control of HIV-1 replication by $\mathrm{CD} 8^{+} \mathrm{T}$ cells is reflected by their avidity, polyfunctionality, and clonal turnover. The Journal of Experimental Medicine. 2007; 204:2473-2485. [PubMed: 17893201]

29. Mendoza D, et al. HLA B*5701 ${ }^{+}$Long-Term Nonprogressors/Elite Controllers are not Distinguished from Progressors by the Clonal Composition of HIV-Specific CD8 ${ }^{+} \mathrm{T}-\mathrm{Cells}$. $\mathrm{J}$ Virol. 2012

30. van Bockel DJ, et al. Persistent Survival of Prevalent Clonotypes within an Immunodominant HIV Gag-Specific CD8 ${ }^{+}$T Cell Response. The Journal of Immunology. 2011; 186:359-371. [PubMed: 21135165]

31. Iglesias MC, et al. Escape from highly effective public $\mathrm{CD}^{+}{ }^{+}$-cell clonotypes by HIV. Blood. 2011; 118:2138-2149. [PubMed: 21734237]

32. Day CL, et al. Proliferative capacity of epitope-specific CD8 T-cell responses is inversely related to viral load in chronic human immunodeficiency virus type 1 infection. J Virol. 2007; 81:434-8. [PubMed: 17050606]

33. Champagne P, et al. Skewed maturation of memory HIV-specific CD8 T lymphocytes. Nature. 2001; 410:106-11. [PubMed: 11242051]

34. Hamann, D.r., et al. Phenotypic and Functional Separation of Memory and Effector Human CD8 ${ }^{+}$ T Cells. The Journal of Experimental Medicine. 1997; 186:1407-1418. [PubMed: 9348298]

35. Chen H, et al. Differential Neutralization of Human Immunodeficiency Virus (HIV) Replication in Autologous CD4 T Cells by HIV-Specific Cytotoxic T Lymphocytes. J. Virol. 2009; 83:31383149. [PubMed: 19158248]

36. Julg B, et al. Infrequent Recovery of HIV from but Robust Exogenous Infection of Activated $\mathrm{CD}^{+}{ }^{+} \mathrm{T}$ Cells in HIV Elite Controllers. Clinical Infectious Diseases. 2010; 51:233-238. [PubMed: 20550452]

37. Brockman MA, Tanzi GO, Walker BD, Allen TM. Use of a novel GFP reporter cell line to examine replication capacity of CXCR4- and CCR5-tropic HIV-1 by flow cytometry. J Virol Methods. 2006; 131:134-42. [PubMed: 16182382]

38. Schneidewind A, et al. Structural and Functional Constraints Limit Options for Cytotoxic TLymphocyte Escape in the Immunodominant HLA-B27-Restricted Epitope in Human Immunodeficiency Virus Type 1 Capsid. J. Virol. 2008; 82:5594-5605. [PubMed: 18385228]

39. Miles JJ, Douek DC, Price DA. Bias in the alphabeta T-cell repertoire: implications for disease pathogenesis and vaccination. Immunol Cell Biol. 2011; 89:375-87. [PubMed: 21301479]

40. Liu D, et al. Integrin-dependent organization and bidirectional vesicular traffic at cytotoxic immune synapses. Immunity. 2009; 31:99-109. [PubMed: 19592272]

41. Stewart-Jones GBE, et al. Crystal structures and KIR3DL1 recognition of three immunodominant viral peptides complexed to HLA-B*2705. European Journal of Immunology. 2005; 35:341-351. [PubMed: 15657948]

42. Price DA, et al. Public clonotype usage identifies protective Gag-specific $\mathrm{CD}^{+} \mathrm{T}$ cell responses in SIV infection. The Journal of Experimental Medicine. 2009; 206:923-936. [PubMed: 19349463]

43. Dong T, et al. HIV-specific cytotoxic T cells from long-term survivors select a unique $\mathrm{T}$ cell receptor. J Exp Med. 2004; 200:1547-57. [PubMed: 15596521]

44. Venturi V, et al. A mechanism for TCR sharing between T cell subsets and individuals revealed by pyrosequencing. J Immunol. 2011; 186:4285-94. [PubMed: 21383244]

45. Miura T, et al. Genetic characterization of human immunodeficiency virus type 1 in elite controllers: lack of gross genetic defects or common amino acid changes. J Virol. 2008; 82:842230. [PubMed: 18562530] 
46. Allen TM, et al. Selection, transmission, and reversion of an antigen-processing cytotoxic Tlymphocyte escape mutation in human immunodeficiency virus type 1 infection. J Virol. 2004; 78:7069-78. [PubMed: 15194783]

47. Walker BD, et al. Long-term culture and fine specificity of human cytotoxic T-lymphocyte clones reactive with human immunodeficiency virus type 1. Proc Natl Acad Sci U S A. 1989; 86:9514-8. [PubMed: 2480604]

48. Varadarajan $\mathrm{N}$, et al. A high-throughput single-cell analysis of human $\mathrm{CD} 8^{+} \mathrm{T}$ cell functions reveals discordance for cytokine secretion and cytolysis. The Journal of Clinical Investigation. 2011; 121:4322-31. [PubMed: 21965332]

49. Yang OO, et al. Impacts of avidity and specificity on the antiviral efficiency of HIV-1-specific CTL. J Immunol. 2003; 171:3718-24. [PubMed: 14500671]

50. Wilson CC, et al. Ex vivo expansion of CD4 lymphocytes from human immunodeficiency virus type 1-infected persons in the presence of combination antiretroviral agents. J Infect Dis. 1995; 172:88-96. [PubMed: 7541065] 
a

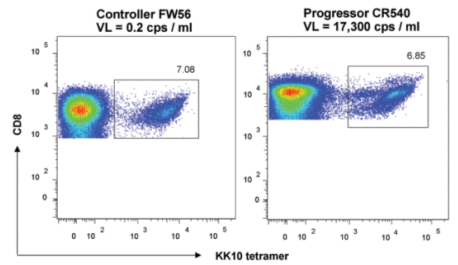

b

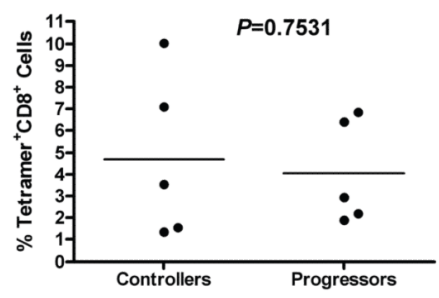

C

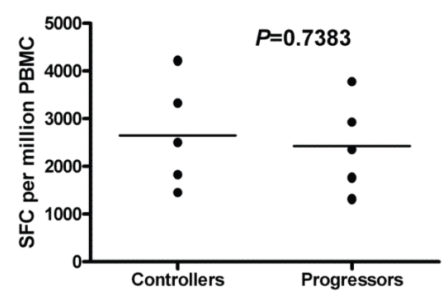

Fig. 1. Quantification of KK10-specific $\mathrm{CD8}^{+} \mathrm{T}$ cell responses (a) Scatterplot of the percentage of HLA-B*27-KK10-specific CD8 ${ }^{+} \mathrm{T}$ cells in a controller (FW56) and a progressor (CR540) determined by flow cytometry and staining with HLA class I tetramers. (b) No significant difference between the controllers $(n=5)$ and progressors $(\mathrm{n}=5)$ in terms of the percentage of KK10 tetramer-positive cells in bulk CD8 ${ }^{+}$ T cells. (c) KK10-specific CD8 ${ }^{+} \mathrm{T}$ cell responses in PBMC were assessed directly ex vivo in an IFN- $\gamma$ ELISPOT assay following KK10 peptide stimulation. There were no significant differences in response magnitude (calculated as spot forming cells (SFC) per million PBMC) between the controllers $(n=5)$ and progressors $(n=5)$. Statistical comparisons were made using the Mann-Whitney test. 
a

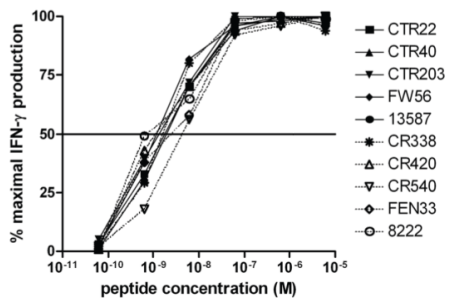

b

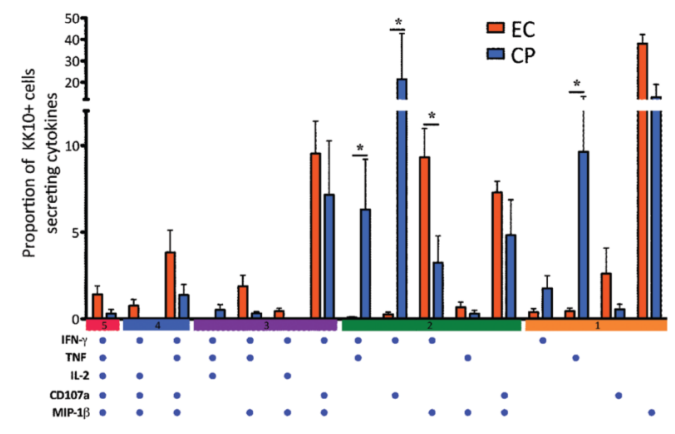

C

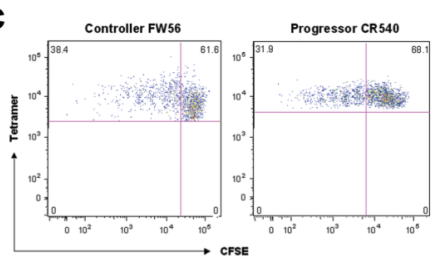

d

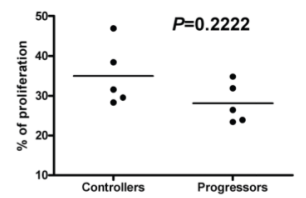

Fig. 2. Functional characteristics of KK10-specific $\mathrm{CD8}^{+} \mathrm{T}$ cells

(a) Functional avidity of KK10-specific $\mathrm{CD}^{+} \mathrm{T}$ cells as measured by peptide titration of PBMC in the IFN- $\gamma$ ELISPOT assay. (b) Expression of IFN- $\gamma$, TNF, IL-2, MIP-1 $\beta$ and CD107a were measured in KK10-specific CD ${ }^{+} \mathrm{T}$ cells from the five HLA-B $* 2705$-positive controllers (EC) and five progressors (CP) following PBMC stimulation with KK10 peptide. The bars represent proportion of subpopulations of KK10-specific cells expressing different combinations of effector functions. The y-axis shows the mean percentage of all cells displaying a particular combination. Statistical comparisons were made using the MannWhitney test; * denotes $\mathrm{p}<0.05$. (c) Scatterplot of proliferation of tetramer-positive KK10specific $\mathrm{CD}^{+} \mathrm{T}$ cells, as shown by CFSE-low cells, at day 7 following stimulation of bulk $\mathrm{CD}^{+} \mathrm{T}$ cells from a controller (FW56) and a progressor (CR540) with virally infected HLA-B*2705-encoding GXR cells. (d) No significant difference was observed in proliferative capacity of KK10-specific $\mathrm{CD}^{+} \mathrm{T}$ cells as measured by CFSE intensity by flow cytometry between the controllers $(n=5)$ and progressors $(n=5)$. 

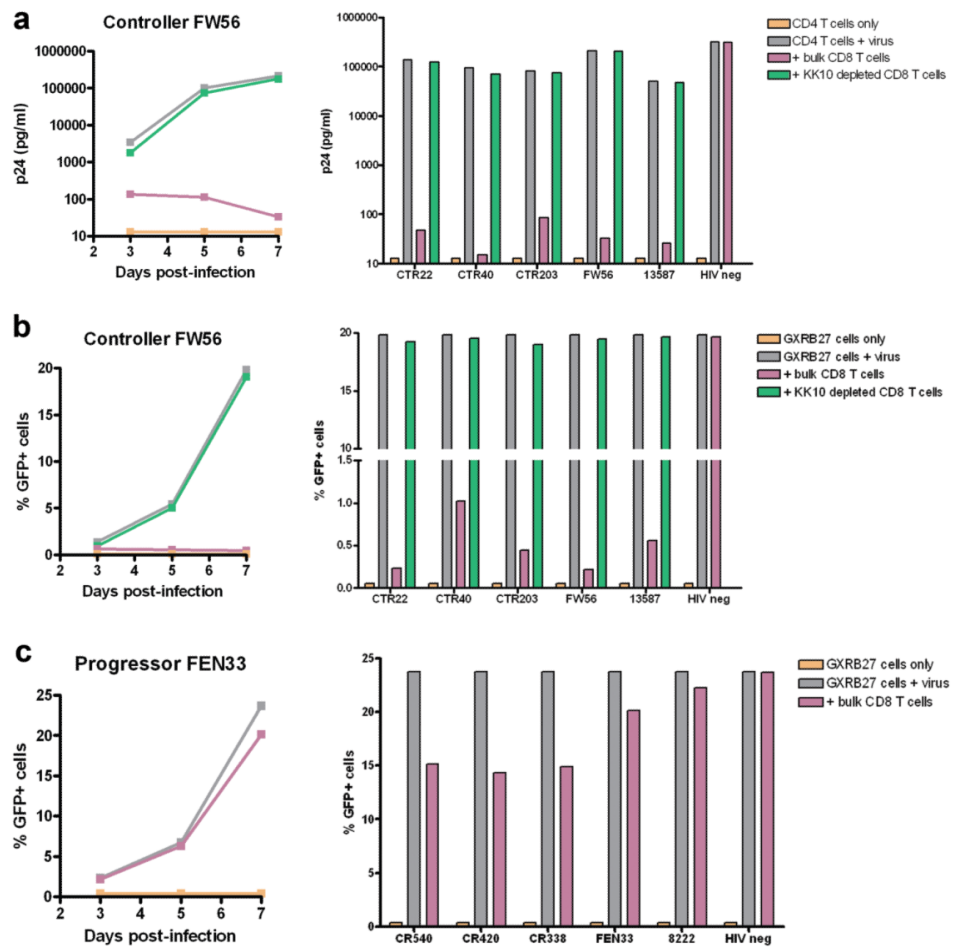

Fig. 3. Virus neutralization by ex vivo KK10-specific $\mathrm{CD8}^{+} \mathrm{T}$ cells

(a) The production of $\mathrm{p} 24$ antigen by autologous $\mathrm{CD}^{+} \mathrm{T}$ cells infected with NL4-3 wildtype virus were evaluated in the presence of bulk $\mathrm{CD}^{+} \mathrm{T}$ cells or KK10-specific celldepleted $\mathrm{CD}^{+} \mathrm{T}$ cells over 7 days for all 5 controllers. Data are shown over 7 days for a representative subject (FW56, left) and for all 5 controllers at day 7 (right). Uninfected $\mathrm{CD} 4^{+} \mathrm{T}$ cells and virally infected $\mathrm{CD} 4^{+} \mathrm{T}$ cells were used as negative and positive controls, respectively. (b) The virus inhibition assay was performed using the HLA-B*2705-encoding GXR cell line and GFP reporter assay. The reporter cells were cultured in the presence of bulk $\mathrm{CD}^{+} \mathrm{T}$ cells or KK10-specific cell-depleted $\mathrm{CD}^{+} \mathrm{T}$ cells over 7 days. The proportions of GFP-positive cells were analyzed by flow cytometry as shown for an representative subject over 7 days (FW56, left) and for all 5 controllers at day 7 (right). (c) Virus replication was evaluated in HLA-B*2705-encoding GFP reporter GXR cells in the presence of bulk $\mathrm{CD}^{+} \mathrm{T}$ cells over 7 days for all 5 progressors. The proportion of GFPpositive cells was analyzed by flow cytometry as shown for an example over 7 days (FEN33, left) and for all 5 progressors at day 7 (right). 
a

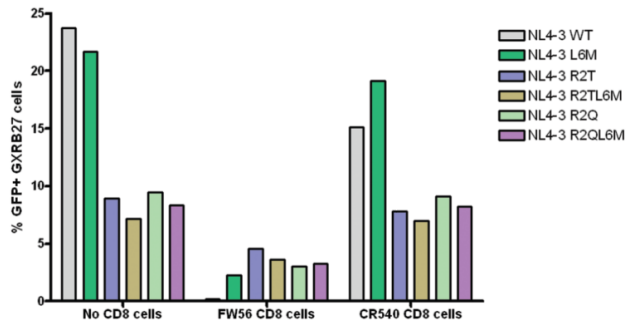

b

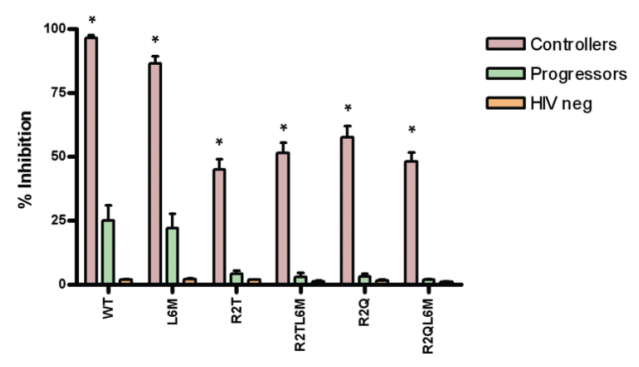

C

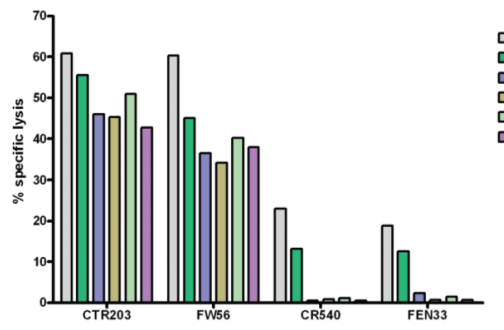

Fig. 4. Recognition of viral variants by KK10-specific $\mathrm{CD8}^{+} \mathrm{T}$ cells

(a) Inhibition of replication of NL4-3 wild-type virus and the designated NL4-3 variants was evaluated in HLA-B*2705-encoding GFP reporter GXR cells in the presence of ex vivo $\mathrm{CD}^{+} \mathrm{T}$ cells isolated from a controller (FW56) and a progressor (CR540) at an effector/ target cell ratio of 1:1. Virus replication was calculated as the proportion of GFP-positive cells by flow cytometry at day 7 in culture. (b) Summary of data from pools of 5 controllers, 5 progressors, and 12 HIV-1 negative individuals demonstrating different antiviral efficacy for $e x$ vivo $\mathrm{CD}^{+} \mathrm{T}$ cells from these groups. Significance was tested with a Mann-Whitney test; * denotes $\mathrm{p}<0.0001$. (c) The ability of ex vivo CD ${ }^{+} \mathrm{T}$ cells from controllers (CTR203 and FW56) and progressors (CR540 and FEN33) to kill live virally infected HLA-B*2705encoding GFP reporter GXR cells was tested in the standard 4-h chromium release assay at an effector/target cell ratio of 10:1. Viable virally infected (GFP-positive) GXR cells were sorted by a FACS Aria cell-sorting instrument after infection for 5 days and used as target cells. 


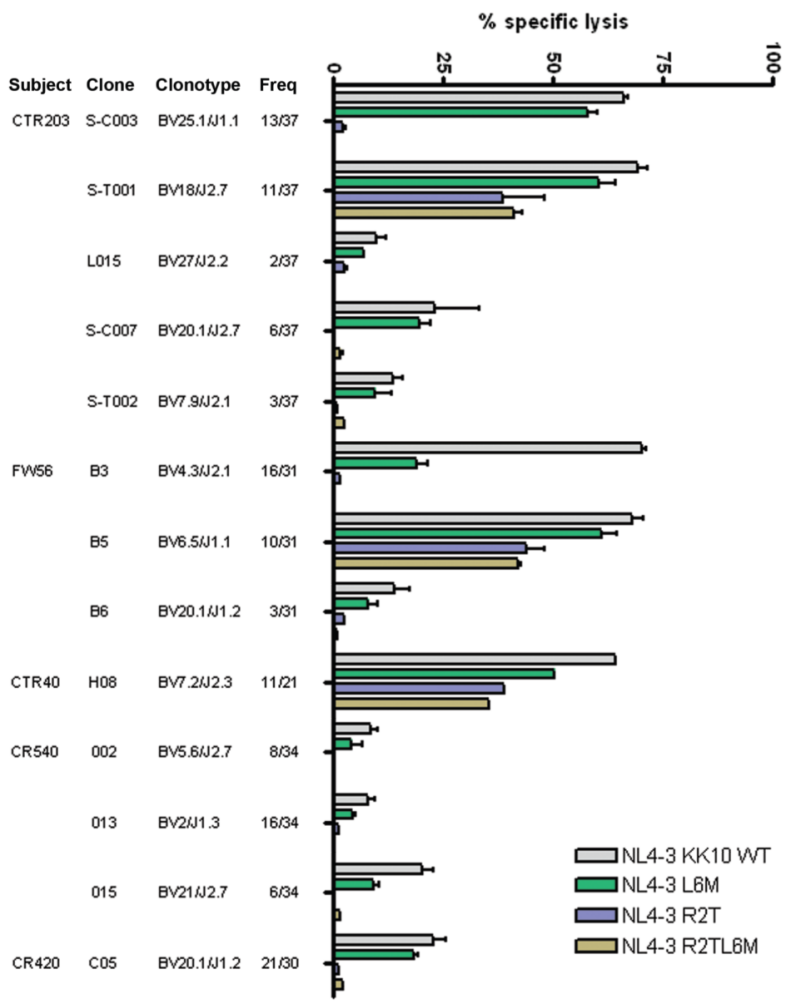

Fig. 5. Differential antiviral efficacy of $B * 27-K K 10$ specific clonotypes

The ability of KK10-specific clonotypes to recognize NL4-3 wild-type and variant viruses was tested in the standard 4-h chromium release assay with virally infected HLA-B*2705encoding GFP reporter GXR cells at an effector/target cell ratio of 1:1. Viable infected (GFP-positive) GXR cells were sorted by a FACS Aria cell-sorting instrument after infection for 5 days and used as target cells. Data are shown for three controllers (CTR203, FW56 and CTR40) and two progressors (CR540 and CR420). 


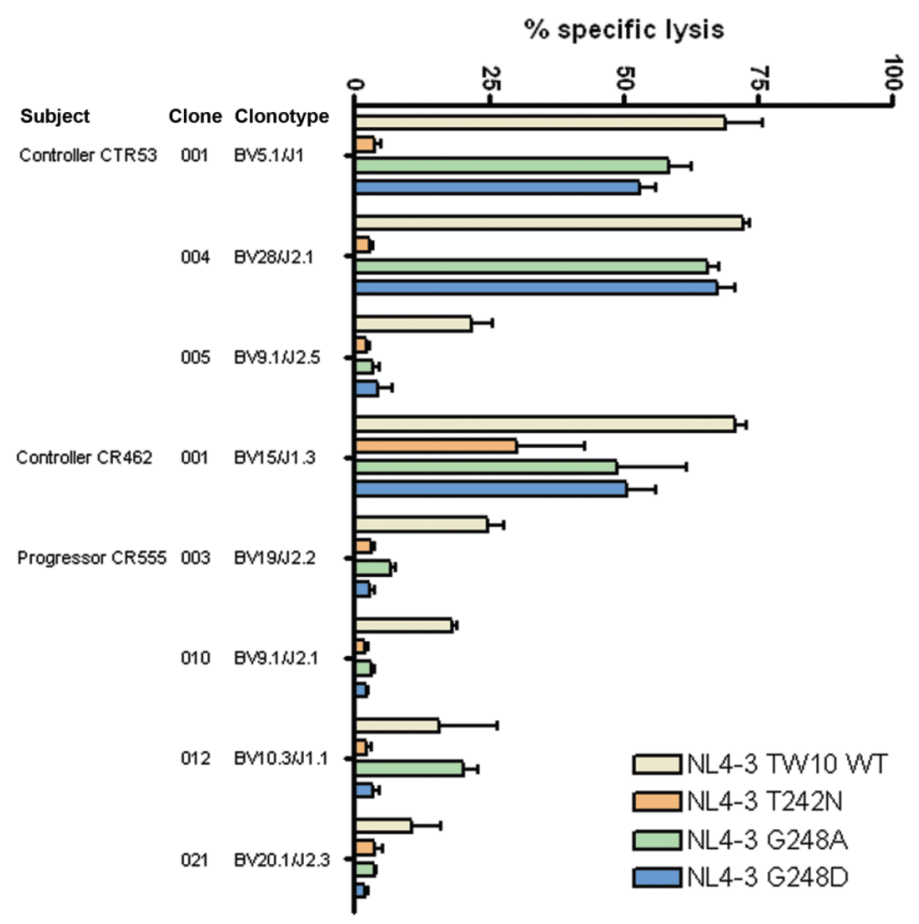

Fig. 6. Differential antiviral efficacy of $B * 57-T W 10$ specific clonotypes The ability of HLA-B*5701 TW10-specific CD8 ${ }^{+}$T cell clones generated from HLA-B*57 positive elite controllers (CTR53, CR462) and a chronic progressor (CR555) to recognize NL4-3 wild-type and variant viruses was tested in the standard 4-h chromium release assay with virally infected HLA-B*5701-encoding GFP reporter GXR cells at an effector/target cell ratio of 1:1. Viable infected (GFP-positive) GXR cells were sorted by a FACS Aria cellsorting instrument after infection for 5 days and used as target cells. 
a
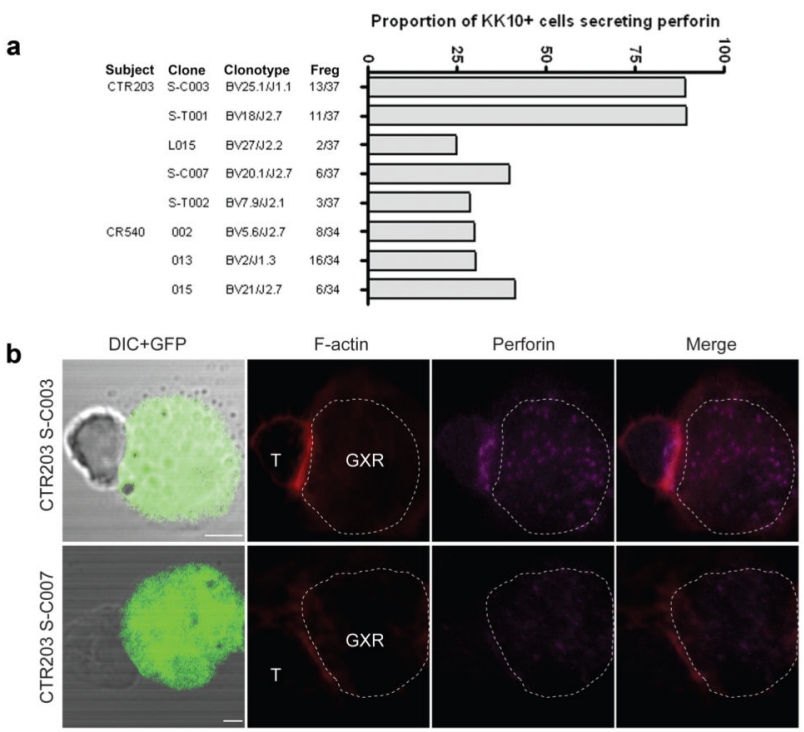

c

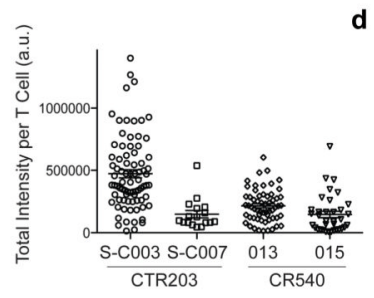

d

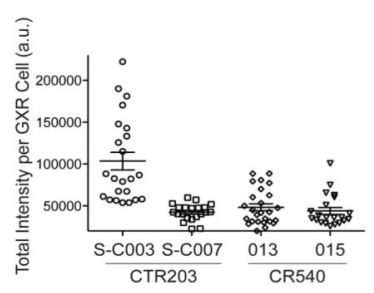

Fig. 7. Differential perforin loading and delivery of clonotypes

(a) Perforin expression of clonotypes was examined by flow cytometry after 3 days of culture with virally infected and uninfected HLA-B*2705-expressing GFP reporter GXR cells. Values indicate percentages of perforin secreting KK10-specific cells upon culture with virally infected target cells after subtracting background from effector cells incubated with uninfected target cells. (b) Differential interference contrast (DIC) images of effector cells with GFP-fluorescing target cells (DIC+GFP) are shown on the Left. Confocal microscope $\mathrm{z}$-series were obtained. Projected serial confocal sections through conjugation between effector cells and HLA-B*2705-encoding GFP reporter GXR cells are shown (Green). F-actin was stained with phalloidin-Alexa Fluor 647 (Red). Perforin was stained with anti-perforin primary Ab followed by Alexa Fluor 568-conjugated secondary mAb (Purple). Merged overlays are on the Right. Clones were imaged $30 \mathrm{~min}$ after incubation with HIV-1 infected GXR cells. The dominant clonotype S-C003 (top) and subdominant clonotype S-C007 (bottom) from controller CTR203 are indicated. Scale bars are $3.0 \mu \mathrm{m}$. (c) Total intensity of perforin per $\mathrm{T}$ cell from representative dominant clonotypes (S-C003 and 013) and subdominant clonotypes (S-C007 and 015) from controller CTR203 and progressor CR540 are shown following exposure to HIV-1 infected GXR cells. (d) Intensity of perforin staining in GXR target cells following exposure to the clonotypes shown in (c). Data for (c) and (d) were obtained from two independent experiments. Error bars indicate the standard error of the mean (SEM). 


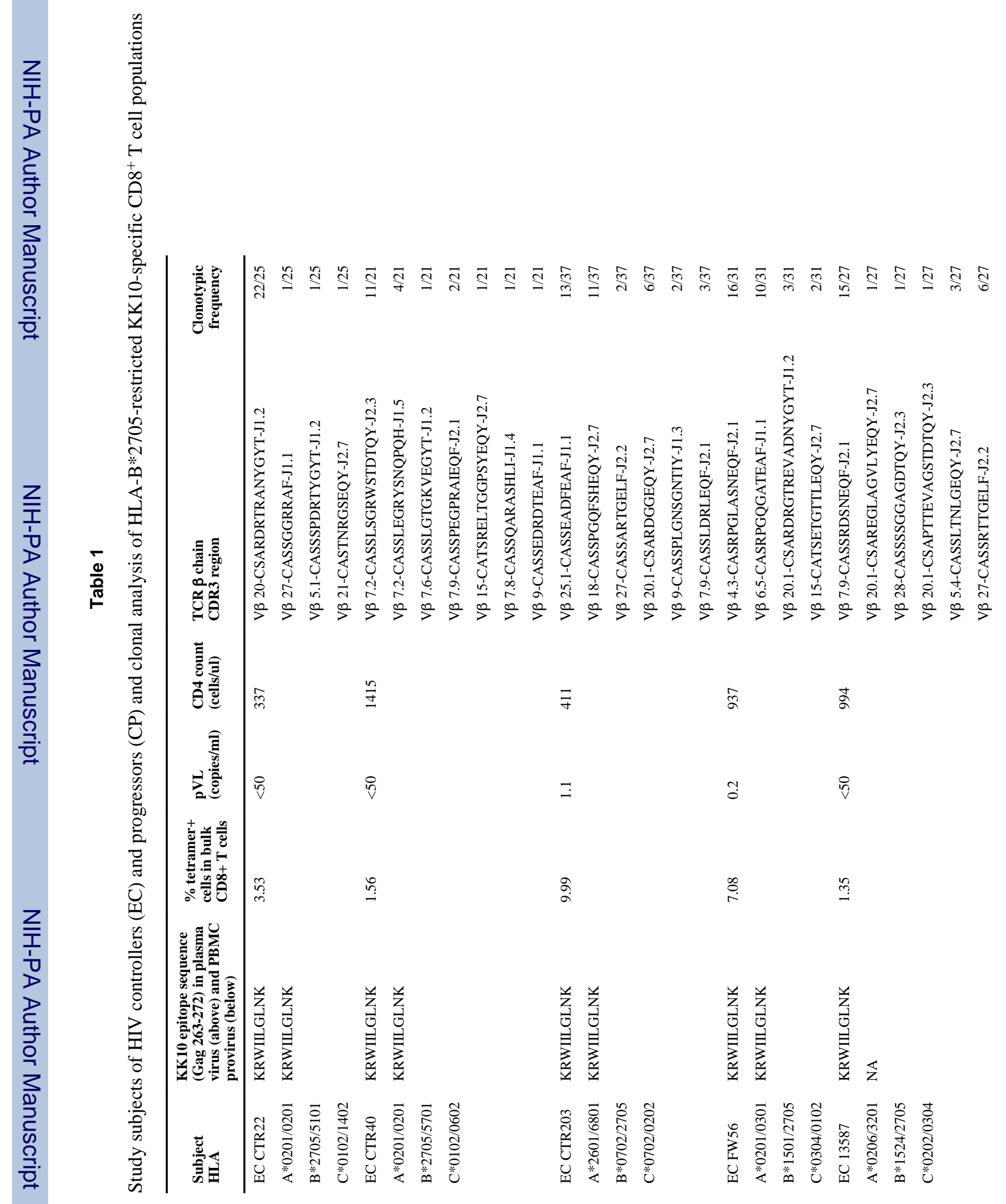




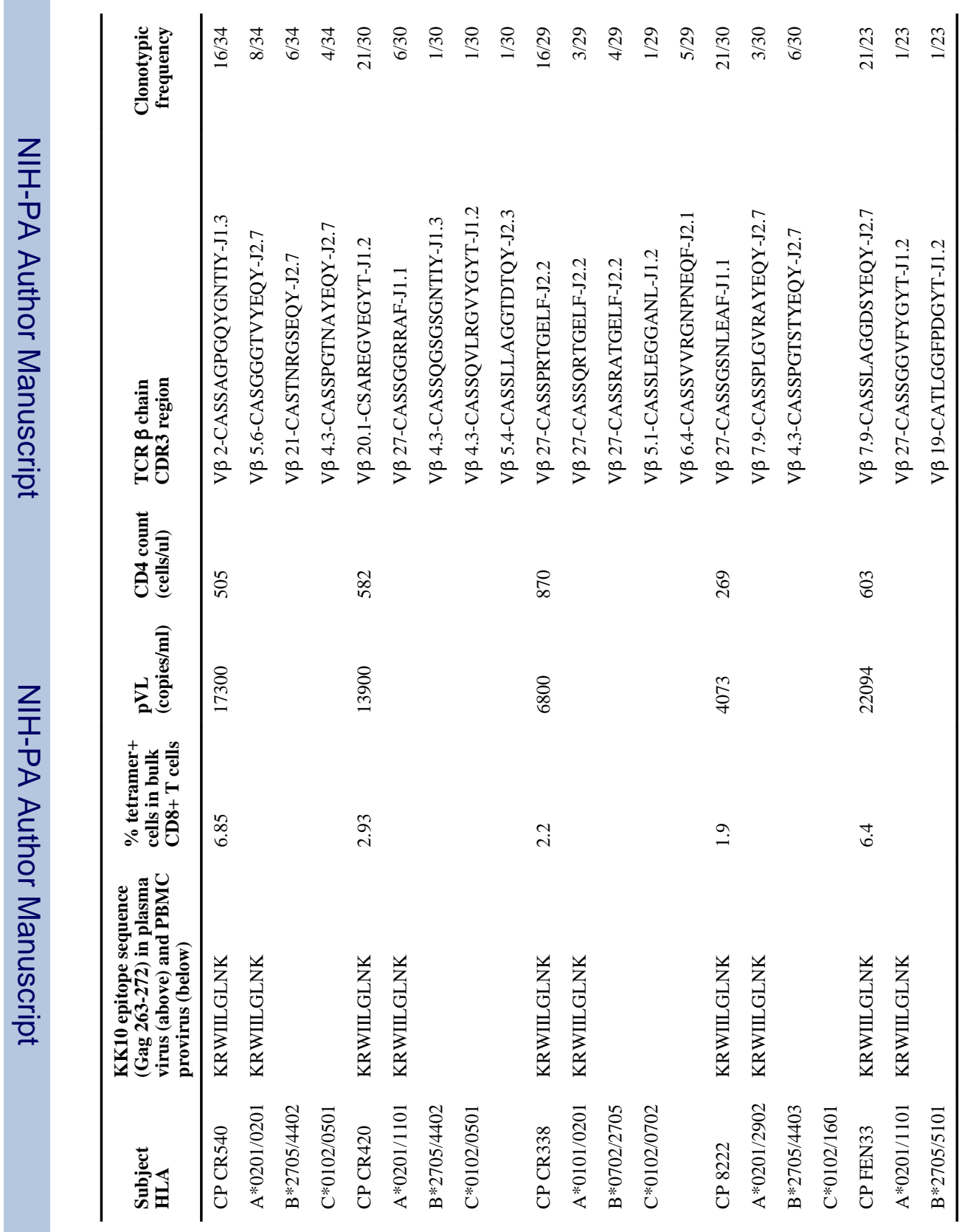

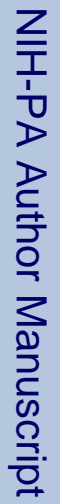




\section{Table 2}

HLA-B*2705-restricted KK10 and HLA-B*57-restricted TW10 epitope sequences in HIV-1 NL4-3 strain and its viral variants

\begin{tabular}{|c|c|}
\hline Viral isolate & Epitope amino acid \\
\hline KK10 (residues 263 to 272) & KRWIILGLNK \\
\hline NL4-3 KK10 WT & $\ldots \ldots \ldots \ldots$ \\
\hline NL4-3 L268M & ........... \\
\hline NL4-3 R264T &. Т......... \\
\hline NL4-3 R264T/L268M &. Т...M.... \\
\hline NL4-3 R264Q & Q......... \\
\hline NL4-3 R264Q/L268M &. $\mathrm{Q} \ldots \mathrm{M} \ldots$ \\
\hline TW10 (residues 240 to 249) & TSTLQEQIGW \\
\hline NL4-3 TW10 WT & $\ldots \ldots \ldots$ \\
\hline NL4-3 T242N &.. $\mathrm{N} \ldots \ldots \ldots$ \\
\hline NL4-3 G248A & …...... \\
\hline NL4-3 G248D & .........D. \\
\hline
\end{tabular}

Nat Immunol. Author manuscript; available in PMC 2013 July 01. 
Table 3

Clonotypes of HLA-B*2705-restricted KK10-specific CD8 ${ }^{+} \mathrm{T}$ cell clones

\begin{tabular}{lllc}
\hline Clone & TCRBV & CDR3 & TCRBJ \\
\hline CTR203 S-C003 & 25.1 & CASSEADFEAF & 1.1 \\
CTR203 S-T001 & 18 & CASSPGQFSHEQY & 2.7 \\
CTR203 L015 & 27 & CASSARTGELF & 2.2 \\
CTR203 S-C007 & 20.1 & CSARDGGEQY & 2.7 \\
CTR203 S-T002 & 7.9 & CASSLDRLEQF & 2.1 \\
FW56 B3 & 4.3 & CASRPGLASNEQF & 2.1 \\
FW56 B5 & 6.5 & CASRPGQGATEAF & 1.1 \\
FW56 B6 & 20.1 & CSARDRGTREVADNYGYT & 1.2 \\
CTR40 H08 & 7.2 & CASSLSGRWSTDTQY & 2.3 \\
CR540 002 & 5.6 & CASGGGTVYEQY & 2.7 \\
CR540 013 & 2 & CASSAGPGQYGNTIY & 1.3 \\
CR540 015 & 21 & CASTNRGSEQY & 2.7 \\
CR420 C0525.1 & 20.1 & CSAREGVEGYT1.1 & 1.2 \\
\hline
\end{tabular}

Nat Immunol. Author manuscript; available in PMC 2013 July 01. 